\title{
Zur Kenntnis der organischen Gerüstsubstanz des Anthozoënskeletts.
}

II. Mitteilung.

Von

Carl Th. Mörner, Upsala.

(Der Redaktion zugegangen am 3. Februar 1908.)

Im Anschluß an eine vorhergehende Mitteilung ${ }^{1}$ ) und unter Hinweis auf die dort gelieferten Angaben über Vorbehandlung des Untersuchungsmaterials, Analysenmethoden u. dgl. berichten wir hiermit über den Fortgang der Untersuchung. Die Gruppe Gorgonacea ist bereits behandelt worden; hier sollen nun die übrigen Anthozoëngruppen, die ein an organischer Gerüstsubstanz reiches Skelett besitzen, behandelt werden, nämlich die zu der Ordnung Alcyonaria gehörigen Gruppen der Pennatulaceen und Alcyonaceen, sowie die zur Ordnung Zoantharia gehörige Gruppe der Antipathideen. Leider ist - ungeachtet aller Bemühungen und alles erwiesenen Entgegenkommens seitens der betreffenden Museums- und Institutsdirektoren $\left.{ }^{2}\right)$ - das Untersuchungsmaterial innerhalb der letztgenannten Gruppen weder qualitativ noch quantitativ genommen, bei weitem nicht so reichlich gewesen, wie betreffs der Gruppe Gorgonacea, welcher Umstand einige Einschränkungen hinsichtlich der analytischen Bearbeitung notwendig gemacht hat.

\section{Pennatulacea. ${ }^{3}$ )}

Die erste Schrift, in der eine Pennatulacee, Pennatula phosphorea L., in chemischer Hinsicht erwähnt wird, dürfte Valenciennes' «Extrait d'une monographie de la famille des

1) Diese Zeitschrift, Bd. LI (1907), S. 33.

2) Für die Freundlichkeit, lediglich zur Förderung dieser Arbeit die Einsammlung von Pennatulaceenmaterial betrieben zu haben, erlaube ich mir an dieser Stelle Herrn Direktor Lic. Phil. Hj. Ös tergren (Christineberg, Bohuslän) verbindlichst zu danken.

$\left.{ }^{8}\right)$ Das Skelett der hierhergehörigen Arten ist als Ekto-, genauer bestimmt als Achsen-Skelett zu bezeichnen. 
Gorgonidées de la classe des Polypes ${ }^{1}$ ) sein. Die Angabe beschränkt sich jedoch auf die Mineralbestandteile des Skeletts (Gehalt an Calciumphosphat in beträchtlicher Menge ${ }^{2}$ ). Die einzige sonstige Pennatulacee, die sich in der chemischen Literatur erwähnt findet, ist Funiculina quadrangularis Pall. Krukenberg ${ }^{3}$ ) glaubt bei Hydrolysierung ihrer organischen Gerüstsubstanz mittelst verdünnter Schwefelsäure Leucin und Glykokoll erhalten zu haben (eine Prüfung auf Halogene ist nicht angestellt worden). Von größerem Interesse für die vorliegende Arbeit ist Drechsels Angabe, ${ }^{4}$ ) daß die organische Gerüstsubstanz des Skeletts der genannten Pennatulacee im Gegensatz zum Gorgonin (aus Gorgonia Cavolini v. Koch) - «nur Spuren von Jod» enthält.

\section{Untersuchungsresultate. ${ }^{5}$ )}

Soweit das aus der angestellten Untersuchung (siehe die Tabelle) hervorgeht, repräsentiert die organische Gerüstsubstanz verschiedener Pennatulaceen einen ziemlich homogenen Typus, charakterisiert durch niedrigen Jod-und Chlor-Gehalt und wesentlich größeren, im ganzen höchst beträchtlichen Bromgehalt. Der Schwefelgehalt stimmt mit dem bei Gorgonaceen angetroffenen überein.

Der halogensubstituierte Proteinstoff, der die organische Gerüstsubstanz der Pennatulaceen bildet - es sei mir erlaubt, ihn als Pennatulin zu bezeichnen - kann nicht identisch mit dem der Gorgonaceen (Gorgonin) sein. Abgesehen von dem Unterschied in bezug auf Konsistenz und Aussehen Pennatulin hat sich stets fast ungefärbt (schwach gelblich) gezeigt, während Gorgonin mehr oder weniger dunkel gefärbt

1) Compt. rend. de l'Académie des Sciences, Bd. XLI (1855), S. 7.

2) Valenciennes' Ausspruch: *Ces axes [de Pennatula phosphorea] sont les seuls produits des animaux rayonnés qui aient avec leur carbonate de chaux une quantité notable de phosphate de chaux», hat sich bei meinen Untersuchungen nicht bestätigt. Auf ein entgegengesetztes Verhältnis ist bereits in der ersten Mitteilung (S. 36) hingewiesen worden.

9) Vergleich.-physiol. Studien, Abteil. 5, Heidelberg 1881, S. 15.

4) Zeitschrift f. Biologie, Bd. XXXIII (1896), S. 96.

5) Hier, wie auch im folgenden, beziehen sich die Prozentzahlen auf aschenfreie Trockensubstanz. 


\begin{tabular}{|c|c|c|c|c|c|c|c|}
\hline \multirow[t]{2}{*}{ Nr. } & \multirow[t]{2}{*}{ Artbezeichnung } & \multirow[t]{2}{*}{ Fundort } & \multirow{2}{*}{$\begin{array}{l}\text { Qualit. } \\
\text { Prüfung } \\
\text { auf } \mathrm{Br}\end{array}$} & \multicolumn{3}{|c|}{$\begin{array}{c}\text { Halogengehalt } \\
\%\end{array}$} & \multirow{2}{*}{$\begin{array}{c}\text { S- } \\
\text { Gehalt } \\
\%\end{array}$} \\
\hline & & & & $\mathbf{J}$ & $\mathrm{Br}$ & $\mathrm{Cl}$ & \\
\hline 1 & $\begin{array}{l}\text { Pennatula } \\
\text { phosphorea L. }\end{array}$ & $\begin{array}{l}\text { Schweden } \\
\text { (Bohuslän) }\end{array}$ & positiv & 0,02 & - & - & - \\
\hline 2 & $\begin{array}{l}\text { Pennatula } \\
\text { borealis Sars }\end{array}$ & Norwegen & • & 0,16 & 0,97 & 0,10 & 1,17 \\
\hline 3 & $\begin{array}{l}\text { Virgularia } \\
\text { mirabilis Lamk }\end{array}$ & $\begin{array}{l}\text { Schweden } \\
\text { (Bohuslän) }\end{array}$ & > & Spur & - & 一 & 一 \\
\hline 4 & $\begin{array}{c}\text { Pavonaria } \\
\text { finmarchica Sars }\end{array}$ & $\begin{array}{l}\text { Norwegen } \\
\text { (Oxfjord) }\end{array}$ & > & 0,20 & - & 一 & - \\
\hline $5 \mathrm{a}$ & $\begin{array}{c}\text { Funiculina } \\
\text { quadrangularis Pall. }\end{array}$ & $\begin{array}{l}\text { Schweden } \\
\text { (Bohuslän) }\end{array}$ & ; & 0,08 & - & - & - \\
\hline b & , & $\begin{array}{c}\text { Norwegen } \\
\text { (Trondhjemfjord) }\end{array}$ & , & 0,13 & 1,89 & 0,13 & - \\
\hline $6 a$ & $\begin{array}{l}\text { Halipteris } \\
\text { Christii K. u. D. }\end{array}$ & $\begin{array}{c}\text { Norwegen } \\
\text { (Sydvaranger) }\end{array}$ & , & 0,20 & 1,51 & 0,11 & $1, \mathbb{4 1}$ \\
\hline b. & , & - & , & 0,21 & 1,38 & 0,08 & - \\
\hline $7 a$ & $\begin{array}{l}\text { Kophobelemnon } \\
\text { stelliferum O. F. M. }\end{array}$ & $\begin{array}{l}\text { Schweden } \\
\text { (Bohuslän) }\end{array}$ & $\triangleright$ & 0,05 & - & - & 一 \\
\hline $\mathbf{b}$ & , & $\begin{array}{c}\text { Dänemark } \\
\text { (Skagen) }\end{array}$ & , & 0,14 & - & - & - \\
\hline 8 & $\begin{array}{c}\text { Umbellula } \\
\text { encrinus (L.) Cuvier }\end{array}$ & Nördl. Eismeer & , & 0,09 & - & - & - \\
\hline
\end{tabular}

ist - weicht Pennatulin in einer wesentlichen Hinsicht von Gorgonin ab. Wie bereits von Krukenberg's) betreffs einer Art (Gorgonia verrucosa Pall.) angegeben und dann von mir bei Prüfung einer größeren Anzahl Arten (mit Substanz sowohl von älteren als auch jüngeren, ja, jüngsten Asten) gefunden worden ist, ist Gorgonin vollkommen unlöslich bei Digestion mit Pepsin-HCl (ebenso wie bei solcher Behandlung mit verd. $\mathrm{HCl}$ allein oder mit Essigsäure). Pennatulin verhält sich ganz anders. Bei Digestion schon mit verd. $\mathrm{HCl}$ oder Essigsäure geschieht partielle Auflösung, während die Substanz anschwillt, bisweilen zu geleeartiger Weichheit; mit Pepsin- $\mathrm{HCl}$ wird fast vollstânndige Auflösung erreicht.

1) Korén und Danielsen.

2) loc. cit., S. 3. 


\section{Alcyonacea. $\left.{ }^{1}\right)$}

Für diese Gruppe fehlt es an jeder Literatur bezüglich der Chemie der organischen Gerüstsubstanz, wenn man nicht Harnacks Bemerkung ${ }^{2}$ ) dahin rechnen will, daß Alcyonium digitatum L. (eine sog. Korkkoralle) in geröstetem Zustande (gleichwie gerösteter Badeschwamm, "Spongia tosta») in älteren Zeiten medizinische Anwendung als Kropfmittel gefunden hat, aus welchem Umstande man auf das wahrscheinliche Vorhandensein eines Jodgehalts schließen könnte. Sehr bescheiden ist auch der Beitrag, der hier geliefert werden kann. Nur von zwei Arten:

1. Parisis laxa Verr. (Japan, Formosa) und

2. Melitodes ochracea Verr. (Manilla) ist Material, zudem der Menge nach spärliches, für. mich erreichbar gewesen.

In beiden Fällen ist $J o d$, wie auch Brom, in der organischen Gerüstsubstanz nachgewiesen worden (der Jodgehalt niedrig, 0,15 bezw. 0,05\%). Eine Schwefelbestimmung hat nicht ausgeführt werden können. Auch ist es mir nicht möglich, mich über die Stellung des hier fraglichen Materials zu den Gorgoninsubstanzen äußern. Seine Unlöslichkeit in Pepsin-HCl unterscheidet es mit Bestimmtheit von den Pennatulinsubstanzen.

\section{Antipathidea. $\left.{ }^{*}\right)$}

Die einzigen Angaben betreffs einer chemischen Untersuchung des organischen Skeletts einer Antipathidee (Antipathes species?) rühren von Krukenberg4) her. Da auch hierbei eine Halogenprüfung unterlassen worden ist, haben sie für die vorliegende Darstellung geringe Bedeutung. Nur so viel sei hier erwähnt, daß K. die Substanz als schwefelfrei und (in Übereinstimmung mit Gorgonia-Substanz) unlöslich, u. a. in Pepsin-HCl, angibt.

1) Innerhalb dieser Gruppe sind die Skelettbildungen vom Mesotypus.

2) Münch. Med. Wochenschr., Bd. XVIII (1896), S. 198.

$\left.{ }^{3}\right)$ Skelettbildungen vom Ekotypus, genauer bestimmt Achsenskelett.

4) loc. cit., S. $3 \mathrm{ff}$. 


\section{Untersuchungsresultate.}

\begin{tabular}{|c|c|c|c|c|c|c|c|}
\hline \multirow[t]{2}{*}{ Nr. } & \multirow[t]{2}{*}{ Artbezeichnung } & \multirow[t]{2}{*}{ Fundort } & \multirow{2}{*}{$\begin{array}{l}\text { Qualit. } \\
\text { Prüfung } \\
\text { auf } \mathrm{Br}\end{array}$} & \multicolumn{3}{|c|}{$\begin{array}{c}\text { Halogengehalt } \\
\quad \%\end{array}$} & \multirow{2}{*}{$\begin{array}{c}\text { S- } \\
\text { Gehalt } \\
\%\end{array}$} \\
\hline & & & & $\mathbf{J}$ & $\mathrm{Br}$ & $\mathrm{Cl}$ & \\
\hline 1 & $\begin{array}{l}\text { Antipathes } \\
\text { arctica Lütken }\end{array}$ & $\begin{array}{r}\text { Grönland } \\
\text { (Skarniut) }\end{array}$ & positiv & 0,02 & - & - & - \\
\hline 2 & $\begin{array}{l}\text { Antipathes } \\
\text { species? }\end{array}$ & 1) & $\Rightarrow$ & 1,79 & 0,38 & 0,42 & 0,37 \\
\hline 3 & $\begin{array}{l}\text { Antipathes } \\
\text { species? }\end{array}$ & - & > & 0,81 & 1,53 & 0,73 & 0,25 \\
\hline 4 & $\begin{array}{l}\text { Cirripathes } \\
\text { spiralis Blainv. }\end{array}$ & - & negativ & 5,45 & - & 0,43 & 0,48 \\
\hline 5 & $\begin{array}{l}\text { Arachnopathes } \\
\text { ericoides Esp. }\end{array}$ & - & $>$ & 6,14 & - & 0,68 & 0,47 \\
\hline
\end{tabular}

Wie aus der Tabelle hervorgeht, sind innerhalb der Gruppe der Antipathideen ganz verschiedene Typen hinsichtlich des Halogengehalts repräsentiert. Bezüglich des Jodgehalts sind die Extreme vertreten: während in Nr.1 dieser Grundstoff wenig. mehr als spurenweise vorhanden ist, wetteifern $\mathrm{Nr}$. 4-5 mit den jodreichsten Gorgonaceen (Gorgonia verrucosa, Cavolini und graminea). In bezug auf Brom ist es höchst bemerkenswert, daß dieser Grundstoff - der nunmehr bei 53 Anthozoënarten (Dubletten einberechnet: 63 Specimina) nachgewiesen worden ist - in zwei Fällen fehlt. ${ }^{2}$ ) Der Chlorgehalt ist hier in jedem untersuchten Falle weit augenfälliger, als das innerhalb der übrigen Anthozoëngruppen der Fall gewesen ist. In entgegengesetzte Richtung geht die Abweichung betreffs des Schwefelgehalts. Zwar ist der der organischen Gerüstsubstanz der Antipathideen zugrunde liegende Proteinstoff nicht schwefelfrei - wie das Krukenberg hat geltend machen wollen -

1) Das Untersuchungsmaterial - ein hinsichtlich der Härte und Farbe ebenholzähnliches Fragment $(10 \mathrm{~cm}$ lang; Gewicht: $58 \mathrm{~g})$ des Hauptstammes - trug die Signatur: «Black coral from Teddah (= Teda in Palästina?)

2) Dieses unerwartete Verhältnis ist durch wiederholte Analysen festgestellt worden.

Hoppe-Seyler's Zeitschrift f. physiol. Chemie. LV. 
wohl aber ist er zu den allerschwefelärmsten unter den Proteinstoffen $\mathrm{zu}$ rechnen.

Abgesehen von dieser Abweichung bezüglich des Schwefelgehalts dürfte die organische Gerüstsubstanz der Antipathideen der der Gorgonaceen (dem Gorgonin) nahe stehen. (Sie ist in Übereinstimmung mit dieser letzteren in Pepsin-HCl unlöslich.)

Zusammenfassung der Ergebnisse(betreffs der Anthozoën im allgemeinen).

1. In allen Fällen sind Halogene, in organischer Bindung vorkommend, in der organischen Gerï̈tsubstanz des Skeletts angetroffen worden. Jod ist in allen Fällen (von Spuren bis gegen 70\%) nachgewiesen worden; Brom in allen Fällen (gefundene Menge: $1 / 4-4 \%$ - ausgenommen bei 2 Antipathideen; Chlor in allen Fällen, wo eine solche Untersuchung angestellt worden ist (in der Regel nur ein oder ein paar Zehntel $\%$, bei den Antipathideen in beträchtlicherer Menge, $1 / 2-3 / 4 \%$ ).

2. Für den früher (bezüglich der Gorgonaceen) ${ }^{1}$ ) aufgestellten Satz betreffs der relativen Konstanz des Halogengehalts bei der Art seiner Unabhängigkeit von dem Klima des Standorts sowie seines Mangels an Korrespondenz mit den physikalischen Eigenschaften der Gerüstsubstanz hat die fortgesetzte Untersuchung weitere Bestätigung geliefert, weshalb er auf die Anthozoën im allgemeinen angewendet werden zu können scheint.

3. Die organische Gerüstsubstanz der Pennatulaceen vorschlagsweise Pennatulin genannt - unterscheidet sich von der der übrigen hier fraglichen Anthozoëngruppen durch ihre Löslichkeit in Pepsin-HCl. Die organische Gerüstsubstanz der Gorgonaceen und der Antipathideen dürfte bis auf weiteres zusammenfassend als Gorgonin zu bezeichnen sein. (Von der organischen Gerüstsubstanz der Alcyonaceen ist noch zu wenig bekannt, um auch nur eine vorläufige Äußerung bezüglich ihrer Rubrizierung zu erlauben.)

4. Der Schwefelgehalt ist - wie zuvor bereits für die Gorgonaceen $^{2}$ ) nachgewiesen worden ist - durchgehends als

1) Diese Zeitschrift, Bd. LI, Mitteil. I, S. 61-62.

s) loc. cit. 
so niedrig ${ }^{1}$ ) befunden worden, daß die organischen Gerüstsubstanze des Anthozoënskeletts nicht in die Gruppe der Keratine eingereiht werden können. Am niedrigsten ist er bei Antipathideen gefunden worden (kaum 1/2\%).

Noch bis vor kurzer Zeit war das Vorkommen von organisch gebundenen Halogenen in animalischen Gerüstsubstanzen nur betreffs der Spongien und einer einzigen Gruppe der Anthozoën, der Gorgonaceen, genauer bekannt. Durch die hier vorgelegte Untersuchungsserie ist die Kenntnis in dieser Hinsicht erweitert worden, indem sie nunmehr alle hier in Betracht kommenden Gruppen der Anthozoën umfaßt.

Es ist jedoch meines Erachtens wahrscheinlich, daß ein derartiges Halogenvorkommen sich als weit allgemeiner bei niederen marinen Tieren erweisen wird, als man bisher angenommen hat. Bei der Untersuchung ganz zufälligerweise disponiblen Materials aus der Gruppe der Anneliden ist nämlich auch dieses als halogenhaltig befunden worden. Zur Untersuchung kamen die sorgfältig entkalkten Wohnröhren von Chaetopterus norvegicus Sars und Hyalinaecia tubicola (Müller) Malmgren. Beide enthielten Jod $(0,22$, bezw. $0,09 \%)$, Brom $(0,18$, bezw. $0,12 \%$ ) nebst Spuren von Chlor.

1) Für die Gruppe Alcyonacea fehlt es uns jedoch gegenwärtig an Kenntnissen in dieser Beziehung. 